\title{
Synthesis of Novel S-Glucosides Containing 5-Methylisoxazole Substituted 1,2,4-Triazole
}

\author{
Shujun Chao and Yingling Wang \\ Department of Chemistry, Xinxiang Medical University, Henan, Xinxiang 453003, China
}

Correspondence should be addressed to Shujun Chao; chaoshujun1979@sina.com

Received 1 April 2012; Accepted 19 May 2012

Academic Editor: Lorenzo Cerretani

Copyright (C) 2013 S. Chao and Y. Wang. This is an open access article distributed under the Creative Commons Attribution License, which permits unrestricted use, distribution, and reproduction in any medium, provided the original work is properly cited.

Nine new S- $\beta$-D-glucosides containing 4-aryl-5-(5-methylisoxazol-3-yl)-1,2,4-triazol-3-thiols have been synthesized by the direct glycosylation of 4-aryl-5-(5-methylisoxazol-3-yl)-1,2,4-triazol-3-thiols with 2,3,4,6-tetra-O-acetyl- $\alpha$-D-glucopyranosyl bromide in ethanol in the presence of potassium hydroxide followed by deacetylation using dry ammonia in dry methanol. All the compounds synthesized have been characterized by their elemental analyses and spectral data.

\section{Introduction}

Much attention has been focused on 1,2,4-triazole derivatives for their broad-spectrum activities, such as antitumor [1], anticonvulsant [2], antifungal [3], herbicidal, and plant growth regulatory activities [4]. Up till now, many 1,2,4triazole derivatives have been synthesized, and some of them have been patented for commercial uses. Similarly, 5methylisoxazole derivatives have also shown biological effects such as antibacterial [5] and phytohormone effects [6].

Recently thioglycosides have received considerable attention, because they are widely employed as biological inhibitors, inducers, and ligands for affinity chromatography for carbohydrate processing-enzymes and proteins $[7,8]$. In 1972, Witkoski et al. synthesized Ribavirin [9] and proved that it not only possesses inhibitory activity against a range of DNA and RNA viruses [10,11] but also displays antitumor activity [12] in mice. After the recognized biological properties of Ribavirin, the synthesis and biological evaluation of N-glucosides [13] and C-glucosides [14] containing 1,2,4triazole have been greatly emphasized, but only a few Sglucosides [15] containing 1,2,4-triazole have been reported. To the best of our knowledge, S-glucosides containing 5methylisoxazole substituted 1,2,4-triazole have not been reported to date. In view of these observations and our interests in the synthesis of biologically active heterocyclic compounds, herein we describe the synthesis of novel
S-glucosides containing 5-methylisoxazole substituted 1,2,4triazole from 4-aryl-5-(5-methylisoxazol-3-yl)-1,2,4-triazol3 -thiols and 2,3,4,6-tetra-O-acetyl- $\alpha$-D-glucopyranosyl bromide (Scheme 1).

\section{Experimental}

Melting points were determined on an X-4 microscopic melting point apparatus and were uncorrected. ${ }^{1} \mathrm{H}$ NMR spectra were determined on a Varian Mercury- $300 \mathrm{MHz}$ spectrometer at room temperature using TMS as internal standard, coupling constants $(J)$ were measured in $\mathrm{Hz}$. Elemental analysis were performed by Elementar Vario EL apparatus. Commercially available reagents were used throughout without further purification unless otherwise stated.

General procedure for Preparation of $3-\mathrm{S}-\left(2^{\prime}, 3^{\prime}, 4^{\prime}, 6^{\prime}-\right.$ tetra-O-acetyl- $\beta$-D-glucopyranosyl)-4-aryl-5-(5-methylisoxazol-3-yl)-1,2,4-triazoles(2a 2i).

To a solution of $\mathrm{KOH}(2 \mathrm{mmol})$ in ethanol $(25 \mathrm{~mL})$ was added 1a-1i $(2 \mathrm{mmol})$. After the mixture was stirred for $30 \mathrm{~min}$ at room temperature, 2,3,4,6-tetra-O-acetyl- $\alpha$-Dglucopyranosyl bromide $(2 \mathrm{mmol})$ was added to the solution. The reaction was stirred for an appropriate time and monitored by TLC until the final conversion. The mixture was filtered and washed with water. The crude product was purified by silica gel column chromatography using 


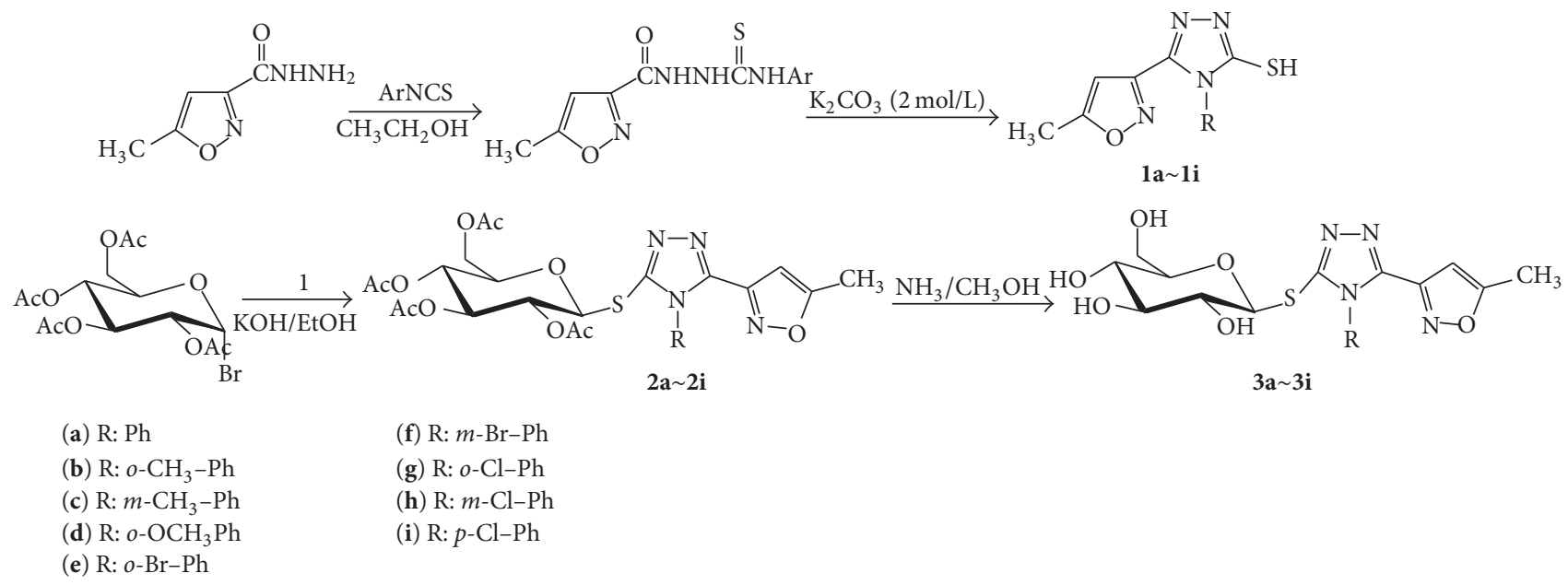

Scheme 1: 4-Aryl-5-(5-methylisoxazol-3-yl)-1,2,4-triazol-3-thiols (1a-1i) were synthesized according to the literature [16, 17].

petroleum ether/ethyl acetate as eluent to afford the pure product.

(2a) Yield 74\%. Mp $172-173^{\circ} \mathrm{C}$. $[\alpha]_{\mathrm{D}} 4^{\circ}\left(\mathrm{c} 1, \mathrm{CH}_{2} \mathrm{Cl}_{2}\right) .{ }^{1} \mathrm{H}$ $\operatorname{NMR}\left(300 \mathrm{MHz}, \mathrm{CDCl}_{3}\right): \delta 1.96\left(\mathrm{~s}, 3 \mathrm{H}, \mathrm{CH}_{3} \mathrm{C}=\mathrm{O}\right)$, $1.97\left(\mathrm{~s}, 3 \mathrm{H}, \mathrm{CH}_{3} \mathrm{C}=\mathrm{O}\right), 1.98\left(\mathrm{~s}, 3 \mathrm{H}, \mathrm{CH}_{3} \mathrm{C}=\mathrm{O}\right), 1.99$ (s, $\left.3 \mathrm{H}, \mathrm{CH}_{3} \mathrm{C}=\mathrm{O}\right), 2.42\left(\mathrm{~s}, 3 \mathrm{H}\right.$, Het- $\left.\mathrm{CH}_{3}\right), 3.82(\mathrm{~m}$, $1 \mathrm{H}), 4.07(\mathrm{dd}, J=12.6,2.1 \mathrm{~Hz}, 1 \mathrm{H}), 4.21(\mathrm{dd}, J=$ $12.6,4.2 \mathrm{~Hz}, 1 \mathrm{H}), 5.04-5.11(\mathrm{~m}, 2 \mathrm{H}), 5.25(\mathrm{t}, J=$ $9.3 \mathrm{~Hz}, 1 \mathrm{H}), 5.57(\mathrm{~d}, J=10.5 \mathrm{~Hz}, 1 \mathrm{H}), 6.55(\mathrm{~s}, 1 \mathrm{H}$, HetH), 6.85-7.51 (m, 5H, ArH). Anal. Calcd. for $\mathrm{C}_{26} \mathrm{H}_{28} \mathrm{~N}_{4} \mathrm{O}_{10} \mathrm{~S}: \mathrm{C}, 53.06 ; \mathrm{H}, 4.79 ; \mathrm{N}, 9.52$. Found: $\mathrm{C}$, $52.89 ; \mathrm{H}, 4.67 ; \mathrm{N}, 9.25$.

(2b) Yield 72\%. Mp 152-154 C. $[\alpha]_{\mathrm{D}} 5^{\circ}\left(\mathrm{c} 1, \mathrm{CH}_{2} \mathrm{Cl}_{2}\right) \cdot{ }^{1} \mathrm{H}$ $\operatorname{NMR}\left(300 \mathrm{MHz}, \mathrm{CDCl}_{3}\right): \delta 1.94\left(\mathrm{~s}, 3 \mathrm{H}, \mathrm{CH}_{3} \mathrm{C}=\mathrm{O}\right)$, $1.96\left(\mathrm{~s}, 3 \mathrm{H}, \mathrm{CH}_{3} \mathrm{C}=\mathrm{O}\right), 1.97\left(\mathrm{~s}, 3 \mathrm{H}, \mathrm{CH}_{3} \mathrm{C}=\mathrm{O}\right), 2.00$ (s, $\left.3 \mathrm{H}, \mathrm{CH}_{3} \mathrm{C}=\mathrm{O}\right), 2.02\left(\mathrm{~s}, 3 \mathrm{H}, \mathrm{CH}_{3}\right), 2.41(\mathrm{~s}, 3 \mathrm{H}$, Het- $\left.\mathrm{CH}_{3}\right), 3.80-3.85(\mathrm{~m}, 1 \mathrm{H}), 4.03-4.14(\mathrm{~m}, 1 \mathrm{H})$, 4.20-4.29 (m, 1H), 5.05-5.14 (m, 2H), 5.25-5.32 (m, $1 \mathrm{H}), 5.64-5.76(\mathrm{~m}, 1 \mathrm{H}), 6.56(\mathrm{~s}, 1 \mathrm{H}, \mathrm{HetH}), 6.87(\mathrm{~d}$, $J=7.5 \mathrm{~Hz}, 1 \mathrm{H}, \mathrm{ArH}), 6.99-7.46$ (m, 3H, ArH). Anal. Calcd. for $\mathrm{C}_{27} \mathrm{H}_{30} \mathrm{~N}_{4} \mathrm{O}_{10} \mathrm{~S}$ : C, 53.81; H, 5.02; N, 9.30. Found: C, 53.45; H, 4.95; N, 8.92 .

(2c) Yield 66\%. Mp 146-147 ${ }^{\circ}$ C. $[\alpha]_{\mathrm{D}} 3^{\circ}\left(\mathrm{c} 1, \mathrm{CH}_{2} \mathrm{Cl}_{2}\right) \cdot{ }^{1} \mathrm{H}$ NMR $\left(300 \mathrm{MHz}, \mathrm{CDCl}_{3}\right): \delta 1.94\left(\mathrm{~s}, 3 \mathrm{H}, \mathrm{CH}_{3} \mathrm{C}=\mathrm{O}\right)$, 1.95 (s, $\left.3 \mathrm{H}, \mathrm{CH}_{3} \mathrm{C}=\mathrm{O}\right), 1.96$ (s, $3 \mathrm{H}, \mathrm{CH}_{3} \mathrm{C}=\mathrm{O}$ ), 1.97 $\left(\mathrm{s}, 3 \mathrm{H}, \mathrm{CH}_{3} \mathrm{C}=\mathrm{O}\right), 2.31\left(\mathrm{~s}, 3 \mathrm{H}, \mathrm{CH}_{3}\right), 2.42(\mathrm{~s}, 3 \mathrm{H}$, Het$\left.\mathrm{CH}_{3}\right), 3.75-3.80(\mathrm{~m}, 1 \mathrm{H}), 4.02-4.06(\mathrm{~m}, 1 \mathrm{H}), 4.21(\mathrm{dd}$, $J=12.9,4.5 \mathrm{~Hz}, 1 \mathrm{H}), 5.02-5.10(\mathrm{~m}, 2 \mathrm{H}), 5.23(\mathrm{t}, J=$ $9.3 \mathrm{~Hz}, 1 \mathrm{H}), 5.57(\mathrm{~d}, J=10.8 \mathrm{~Hz}, 1 \mathrm{H}), 6.54(\mathrm{~s}, 1 \mathrm{H}$, HetH), 6.86 (d, $J=7.8 \mathrm{~Hz}, 1 \mathrm{H}, \mathrm{ArH}), 6.94-7.47(\mathrm{~m}$, $3 \mathrm{H}, \mathrm{ArH}$ ). Anal. Calcd. for $\mathrm{C}_{27} \mathrm{H}_{30} \mathrm{~N}_{4} \mathrm{O}_{10} \mathrm{~S}: \mathrm{C}, 53.81$; H, 5.02; N, 9.30. Found: C, 53.66; H, 5.12; N, 9.07.

(2d) Yield 78\%. Mp 88-90 ${ }^{\circ} \mathrm{C} .[\alpha]_{\mathrm{D}}-9^{\circ}\left(\mathrm{c} 1, \mathrm{CH}_{2} \mathrm{Cl}_{2}\right) \cdot{ }^{1} \mathrm{H}$ $\operatorname{NMR}\left(300 \mathrm{MHz}, \mathrm{CDCl}_{3}\right): \delta 1.99\left(\mathrm{~s}, 3 \mathrm{H}, \mathrm{CH}_{3} \mathrm{C}=\mathrm{O}\right)$, $2.00\left(\mathrm{~s}, 3 \mathrm{H}, \mathrm{CH}_{3} \mathrm{C}=\mathrm{O}\right), 2.01\left(\mathrm{~s}, 3 \mathrm{H}, \mathrm{CH}_{3} \mathrm{C}=\mathrm{O}\right)$, $2.03\left(\mathrm{~s}, 3 \mathrm{H}, \mathrm{CH}_{3} \mathrm{C}=\mathrm{O}\right), 2.41$ (s, 3H, Het- $\left.\mathrm{CH}_{3}\right), 3.65$ $\left(\mathrm{s}, 3 \mathrm{H}, \mathrm{CH}_{3} \mathrm{O}\right), 3.75-3.82(\mathrm{~m}, 1 \mathrm{H}), 4.02-4.10(\mathrm{~m}, 1 \mathrm{H})$,
4.20-4.30 (m, 1H), 5.03-5.14 (m, 2H), 5.22-5.30 (m, $1 \mathrm{H}), 5.53-5.58(\mathrm{~m}, 1 \mathrm{H}), 6.56(\mathrm{~s}, 1 \mathrm{H}, \mathrm{HetH}), 6.87(\mathrm{~d}$, $J=6.9 \mathrm{~Hz}, 1 \mathrm{H}, \mathrm{ArH}), 6.96-7.49(\mathrm{~m}, 3 \mathrm{H}, \mathrm{ArH})$. Anal. Calcd. for $\mathrm{C}_{27} \mathrm{H}_{30} \mathrm{~N}_{4} \mathrm{O}_{11}$ S: C, 52.42; H, 4.89; N, 9.06. Found: C, 52.60; H, 4.93; N, 9.31.

(2e) Yield 64\%. Mp 86-88 ${ }^{\circ} \mathrm{C} .[\alpha]_{\mathrm{D}}-5^{\circ}\left(\mathrm{c} 1, \mathrm{CH}_{2} \mathrm{Cl}_{2}\right) \cdot{ }^{1} \mathrm{H}$ NMR (300 MHz, $\left.\mathrm{CDCl}_{3}\right): \delta 1.99\left(\mathrm{~s}, 3 \mathrm{H}, \mathrm{CH}_{3} \mathrm{C}=\mathrm{O}\right)$, $2.00\left(\mathrm{~s}, 3 \mathrm{H}, \mathrm{CH}_{3} \mathrm{C}=\mathrm{O}\right), 2.02\left(\mathrm{~s}, 3 \mathrm{H}, \mathrm{CH}_{3} \mathrm{C}=\mathrm{O}\right), 2.04$ (s, $\left.3 \mathrm{H}, \mathrm{CH}_{3} \mathrm{C}=\mathrm{O}\right), 2.41\left(\mathrm{~s}, 3 \mathrm{H}\right.$, Het- $\left.\mathrm{CH}_{3}\right), 3.78-3.82(\mathrm{~m}$, $1 \mathrm{H}), 4.03-4.13(\mathrm{~m}, 1 \mathrm{H}), 4.22-4.27(\mathrm{~m}, 1 \mathrm{H}), 5.03-5.17$ $(\mathrm{m}, 2 \mathrm{H}), 5.27(\mathrm{t}, J=9.3 \mathrm{~Hz}, 1 \mathrm{H}), 5.58(\mathrm{~d}, J=9.9 \mathrm{~Hz}$, $1 \mathrm{H}), 6.63$ (s, 1H, HetH), 6.87-7.47 (m, 4H, ArH). Anal. Calcd. for $\mathrm{C}_{26} \mathrm{H}_{27} \mathrm{BrN}_{4} \mathrm{O}_{10} \mathrm{~S}$ : C, 46.78; H, 4.08; N, 8.39. Found: C, $46.48 ; \mathrm{H}, 3.97 ; \mathrm{N}, 8.51$.

(2f) Yield $55 \%$. Mp $98-100^{\circ} \mathrm{C}$. $[\alpha]_{\mathrm{D}} 3^{\circ}\left(\mathrm{c} 1, \mathrm{CH}_{2} \mathrm{Cl}_{2}\right) \cdot{ }^{1} \mathrm{H}$ $\operatorname{NMR}\left(300 \mathrm{MHz}, \mathrm{CDCl}_{3}\right): \delta 2.00\left(\mathrm{~s}, 3 \mathrm{H}, \mathrm{CH}_{3} \mathrm{C}=\mathrm{O}\right)$, $2.02\left(\mathrm{~s}, 3 \mathrm{H}, \mathrm{CH}_{3} \mathrm{C}=\mathrm{O}\right), 2.04\left(\mathrm{~s}, 3 \mathrm{H}, \mathrm{CH}_{3} \mathrm{C}=\mathrm{O}\right), 2.05$ (s, $3 \mathrm{H}, \mathrm{CH}_{3} \mathrm{C}=\mathrm{O}$ ), 2.44 (s, 3H, Het- $\left.\mathrm{CH}_{3}\right), 3.77-3.82$ $(\mathrm{m}, 1 \mathrm{H}), 4.08-4.13(\mathrm{~m}, 1 \mathrm{H}), 4.24(\mathrm{dd}, J=12.9,4.8 \mathrm{~Hz}$, $1 \mathrm{H}), 5.07-5.16(\mathrm{~m}, 2 \mathrm{H}), 5.28(\mathrm{t}, J=9.3 \mathrm{~Hz}, 1 \mathrm{H}), 5.54$ $(\mathrm{d}, J=10.5 \mathrm{~Hz}, 1 \mathrm{H}), 6.60(\mathrm{~s}, 1 \mathrm{H}, \mathrm{HetH}), 6.84-7.51$ (m, $4 \mathrm{H}, \mathrm{ArH}$ ). Anal. Calcd. for $\mathrm{C}_{26} \mathrm{H}_{27} \mathrm{BrN}_{4} \mathrm{O}_{10} \mathrm{~S}$ : C, 46.78; H, 4.08; N, 8.39. Found: C, 46.45; H, 3.83; N, 8.20 .

(2g) Yield 62\%. Mp 90-92 ${ }^{\circ} \mathrm{C} .[\alpha]_{\mathrm{D}}-6^{\circ}\left(\mathrm{c} 1, \mathrm{CH}_{2} \mathrm{Cl}_{2}\right) \cdot{ }^{1} \mathrm{H}$ $\operatorname{NMR}\left(300 \mathrm{MHz}, \mathrm{CDCl}_{3}\right): \delta 1.95\left(\mathrm{~s}, 3 \mathrm{H}, \mathrm{CH}_{3} \mathrm{C}=\mathrm{O}\right)$, $1.97\left(\mathrm{~s}, 3 \mathrm{H}, \mathrm{CH}_{3} \mathrm{C}=\mathrm{O}\right), 1.98\left(\mathrm{~s}, 3 \mathrm{H}, \mathrm{CH}_{3} \mathrm{C}=\mathrm{O}\right), 2.00(\mathrm{~s}$, $\left.3 \mathrm{H}, \mathrm{CH}_{3} \mathrm{C}=\mathrm{O}\right), 2.42\left(\mathrm{~s}, 3 \mathrm{H}\right.$, Het- $\left.\mathrm{CH}_{3}\right), 3.75-3.80(\mathrm{~m}$, $1 \mathrm{H}), 3.99-4.09(\mathrm{~m}, 1 \mathrm{H}), 4.17-4.22(\mathrm{~m}, 1 \mathrm{H}), 4.99-5.12$ $(\mathrm{m}, 2 \mathrm{H}), 5.24(\mathrm{t}, J=9.0 \mathrm{~Hz}, 1 \mathrm{H}), 5.49-5.55(\mathrm{~m}, 1 \mathrm{H})$, $6.64(\mathrm{~s}, 1 \mathrm{H}, \mathrm{HetH}), 6.86-7.54(\mathrm{~m}, 4 \mathrm{H}, \mathrm{ArH})$. Anal. Calcd. for $\mathrm{C}_{26} \mathrm{H}_{27} \mathrm{ClN}_{4} \mathrm{O}_{10} \mathrm{~S}$ : C, 50.12; $\mathrm{H}, 4.37 ; \mathrm{N}$, 8.99. Found: C, 49.75; H, 4.10; N, 8.65 .

(2h) Yield 59\%. Mp 157-158 ${ }^{\circ} \mathrm{C} .[\alpha]_{\mathrm{D}} 1^{\circ}\left(\mathrm{c} 1, \mathrm{CH}_{2} \mathrm{Cl}_{2}\right) .{ }^{1} \mathrm{H}$ $\operatorname{NMR}\left(300 \mathrm{MHz}, \mathrm{CDCl}_{3}\right): \delta 1.98\left(\mathrm{~s}, 3 \mathrm{H}, \mathrm{CH}_{3} \mathrm{C}=\mathrm{O}\right)$, $2.00\left(\mathrm{~s}, 3 \mathrm{H}, \mathrm{CH}_{3} \mathrm{C}=\mathrm{O}\right), 2.01\left(\mathrm{~s}, 3 \mathrm{H}, \mathrm{CH}_{3} \mathrm{C}=\mathrm{O}\right), 2.03$ (s, $3 \mathrm{H}, \mathrm{CH}_{3} \mathrm{C}=\mathrm{O}$ ), 2.43 (s, 3H, Het- $\mathrm{CH}_{3}$ ), 3.75-3.80 
$(\mathrm{m}, 1 \mathrm{H}), 4.09(\mathrm{dd}, J=12.3,1.8 \mathrm{~Hz}, 1 \mathrm{H}), 4.22(\mathrm{dd}$, $J=12.3,4.5 \mathrm{~Hz}, 1 \mathrm{H}), 5.04-5.14(\mathrm{~m}, 2 \mathrm{H}), 5.26(\mathrm{t}$, $J=9.0 \mathrm{~Hz}, 1 \mathrm{H}), 5.52(\mathrm{~d}, J=9.9 \mathrm{~Hz}, 1 \mathrm{H}), 6.61(\mathrm{~s}$, $1 \mathrm{H}, \mathrm{HetH}), 6.86$ (d, J = 8.1 Hz, 1H, ArH), 7.05-7.63 (m, 3H, ArH). Anal. Calcd. for $\mathrm{C}_{26} \mathrm{H}_{27} \mathrm{ClN}_{4} \mathrm{O}_{10} \mathrm{~S}$ : C, 50.12; H, 4.37; N, 8.99. Found: C, 49.91; H, 4.21; N, 8.66.

(2i) Yield 54\%. Mp $170-171^{\circ} \mathrm{C} .[\alpha]_{\mathrm{D}} 1^{\circ}\left(\mathrm{c} 1, \mathrm{CH}_{2} \mathrm{Cl}_{2}\right) \cdot{ }^{1} \mathrm{H}$ NMR $\left(300 \mathrm{MHz}, \mathrm{CDCl}_{3}\right): \delta 2.00\left(\mathrm{~s}, 3 \mathrm{H}, \mathrm{CH}_{3} \mathrm{C}=\mathrm{O}\right)$, $2.01\left(\mathrm{~s}, 3 \mathrm{H}, \mathrm{CH}_{3} \mathrm{C}=\mathrm{O}\right), 2.02\left(\mathrm{~s}, 3 \mathrm{H}, \mathrm{CH}_{3} \mathrm{C}=\mathrm{O}\right), 2.03$ (s, 3H, $\left.\mathrm{CH}_{3} \mathrm{C}=\mathrm{O}\right), 2.43$ (s, 3H, Het- $\left.\mathrm{CH}_{3}\right), 3.77-3.83$ $(\mathrm{m}, 1 \mathrm{H}), 4.08-4.12(\mathrm{~m}, 1 \mathrm{H}), 4.23(\mathrm{dd}, J=12.3,4.8 \mathrm{~Hz}$, $1 \mathrm{H}), 5.07-5.15(\mathrm{~m}, 2 \mathrm{H}), 5.28(\mathrm{t}, J=9.0 \mathrm{~Hz}, 1 \mathrm{H}), 5.57$ $(\mathrm{d}, J=10.2 \mathrm{~Hz}, 1 \mathrm{H}), 6.60(\mathrm{~s}, 1 \mathrm{H}, \mathrm{HetH}), 6.88(\mathrm{~d}, J$ $=7.5 \mathrm{~Hz}, 1 \mathrm{H}, \mathrm{ArH}), 7.05-7.46(\mathrm{~m}, 3 \mathrm{H}, \mathrm{ArH})$. Anal. Calcd. for $\mathrm{C}_{26} \mathrm{H}_{27} \mathrm{ClN}_{4} \mathrm{O}_{10} \mathrm{~S}$ : C, 50.12; H, 4.37; N, 8.99. Found: C, 49.88; $\mathrm{H}, 4.06 ; \mathrm{N}, 8.73$.

General procedure for Preparation of $3-S-\left(2^{\prime}, 3^{\prime}, 4^{\prime}, 6^{\prime}\right.$-tetrahydroxy- $\beta$-D-glucopyranosyl)-4-aryl-5-(5-methylisoxazol3-yl)-1,2,4-triazoles (3a $3 \mathbf{i})$.

Dry gaseous ammonia was passed at $0^{\circ} \mathrm{C}$ for about $1 \mathrm{~h}$ into a solution of $\mathbf{2 a} \sim \mathbf{2} \mathbf{i}(1 \mathrm{mmol})$ in dry $\mathrm{MeOH}$. Then, the mixture was stirred at $25^{\circ} \mathrm{C}$, and the reaction was monitored by TLC. The solution was concentrated, and the analytical pure product was obtained directly by recrystallization from methanol or methanol-petroleum ether.

(3a) Yield 91.5\%. Mp 134-135 C. $[\alpha]_{\mathrm{D}}-46^{\circ}$ (c 1, $\mathrm{CH}_{3} \mathrm{OH}$ ). ${ }^{1} \mathrm{H}$ NMR $\left(300 \mathrm{MHz}, \mathrm{D}_{2} \mathrm{O}\right): \delta 2.36\left(\mathrm{~s}, 6 \mathrm{H}, \mathrm{Ph}-\mathrm{CH}_{3}\right.$ and Het $\left.-\mathrm{CH}_{3}\right), 3.29-3.39(\mathrm{~m}, 3 \mathrm{H}), 3.41-3.47(\mathrm{~m}$, $1 \mathrm{H}), 3.57-3.63(\mathrm{~m}, 1 \mathrm{H}), 3.76(\mathrm{~d}, J=12.3 \mathrm{~Hz}, 1 \mathrm{H})$, 4.83-4.86 (m, 1H), 6.33 (s, 1H, HetH), 6.99-7.53 (m, $5 \mathrm{H}, \mathrm{ArH}$ ). Anal. Calcd. for $\mathrm{C}_{18} \mathrm{H}_{20} \mathrm{~N}_{4} \mathrm{O}_{6} \mathrm{~S}$ : C, 51.42; H, 4.79; N, 13.33. Found: C, 51.14; H, 4.66; N, 12.94.

(3b) : Yield $85 \%$. Mp $122-123^{\circ} \mathrm{C} .[\alpha]_{\mathrm{D}}-45^{\circ}$ (c 1, $\left.\mathrm{CH}_{3} \mathrm{OH}\right)$. ${ }^{1} \mathrm{H}$ NMR $\left(300 \mathrm{MHz}, \mathrm{D}_{2} \mathrm{O}\right): \delta 1.92\left(\mathrm{~s}, 3 \mathrm{H}, \mathrm{Ar}-\mathrm{CH}_{3}\right)$, $2.36\left(\mathrm{~s}, 3 \mathrm{H}, \mathrm{Het}-\mathrm{CH}_{3}\right), 3.29-3.37(\mathrm{~m}, 4 \mathrm{H}), 3.60-3.66$ $(\mathrm{m}, 1 \mathrm{H}), 3.76(\mathrm{t}, J=12.3 \mathrm{~Hz}, 1 \mathrm{H}), 4.95-4.99(\mathrm{~m}, 1 \mathrm{H})$, 6.34 (s, 1H, HetH), 7.00-7.20 (m, 4H, ArH). Anal. Calcd. for $\mathrm{C}_{19} \mathrm{H}_{22} \mathrm{~N}_{4} \mathrm{O}_{6} \mathrm{~S}$ : C, 52.52; H, 5.10; N, 12.90 . Found: C, 52.29; H, 5.01; N, 12.56.

(3c) Yield 94\%. Mp $148-150^{\circ}$ C. $[\alpha]_{D}-44^{\circ}$ (c 1, $\mathrm{CH}_{3} \mathrm{OH}$ ). ${ }^{1} \mathrm{H}$ NMR $\left(300 \mathrm{MHz}, \mathrm{D}_{2} \mathrm{O}\right): \delta 2.36\left(\mathrm{~s}, 6 \mathrm{H}\right.$, Het- $\left.\mathrm{CH}_{3}\right)$, $3.25-3.35(\mathrm{~m}, 3 \mathrm{H}), 3.41-3.47(\mathrm{~m}, 1 \mathrm{H}), 3.55-3.60(\mathrm{~m}$, $1 \mathrm{H}), 3.72(\mathrm{~d}, J=11.7 \mathrm{~Hz}, 1 \mathrm{H}), 4.87(\mathrm{~d}, J=9.9 \mathrm{~Hz}, 1 \mathrm{H})$, 6.31 (s, 1H, HetH), 6.96-7.56 (m, 4H, ArH). Anal. Calcd. for $\mathrm{C}_{19} \mathrm{H}_{22} \mathrm{~N}_{4} \mathrm{O}_{6} \mathrm{~S}$ : C, 52.52; H, 5.10; N, 12.90 . Found: C, 52.34; H, 5.02; N, 12.77.

(3d) Yield $89 \%$. Mp $125-127^{\circ}$ C. $[\alpha]_{D}-48^{\circ}$ (c 1, $\left.\mathrm{CH}_{3} \mathrm{OH}\right)$. ${ }^{1} \mathrm{H}$ NMR $\left(300 \mathrm{MHz}, \mathrm{D}_{2} \mathrm{O}\right): \delta 2.34\left(\mathrm{~s}, 3 \mathrm{H}\right.$, Het- $\left.\mathrm{CH}_{3}\right)$, 3.24-3.48 (m, 7H), 3.57-3.63 (m, $1 \mathrm{H}), 3.68(\mathrm{~s}, 3 \mathrm{H}$, $\left.\mathrm{CH}_{3} \mathrm{O}\right), 3.70-3.76(\mathrm{~m}, 1 \mathrm{H}), 4.83-4.90(\mathrm{~m}, 1 \mathrm{H}), 6.34$ (s, 1H, HetH), 6.96-7.54 (m, 4H, ArH). Anal. Calcd. for $\mathrm{C}_{19} \mathrm{H}_{22} \mathrm{~N}_{4} \mathrm{O}_{7} \mathrm{~S}$ : C, 50.66; $\mathrm{H}, 4.92 ; \mathrm{N}, 12.44$. Found: C, 50.38; H, 5.04; N, 12.11 .

(3e) Yield 93\%. Mp $145-147^{\circ} \mathrm{C} .[\alpha]_{\mathrm{D}}-57^{\circ}$ (c 1, $\mathrm{CH}_{3} \mathrm{OH}$ ). ${ }^{1} \mathrm{H}$ NMR $\left(300 \mathrm{MHz}, \mathrm{D}_{2} \mathrm{O}\right): \delta 2.36\left(\mathrm{~s}, 3 \mathrm{H}, \mathrm{Het}-\mathrm{CH}_{3}\right)$,
3.26-3.44 (m, 4H), 3.57-3.62 (m, 1H), 3.69-3.76 (m, $1 \mathrm{H}), 6.42(\mathrm{~s}, 1 \mathrm{H}, \mathrm{HetH}), 6.99-7.71(\mathrm{~m}, 4 \mathrm{H}, \mathrm{ArH})$. Anal. Calcd. for $\mathrm{C}_{18} \mathrm{H}_{19} \mathrm{BrN}_{4} \mathrm{O}_{6} \mathrm{~S}$ : C, 43.30; H, 3.84; N, 11.22. Found: C, 43.48; H, 4.15; N, 11.03.

(3f) Yield 91\%. Mp 140-142 ${ }^{\circ} \mathrm{C} .[\alpha]_{\mathrm{D}}-29^{\circ}$ (c 1, $\mathrm{CH}_{3} \mathrm{OH}$ ). ${ }^{1} \mathrm{H}$ NMR (300 MHz, $\left.\mathrm{D}_{2} \mathrm{O}\right): \delta 2.35$ (s, 3H, Het- $\mathrm{CH}_{3}$ ), $3.29-3.39(\mathrm{~m}, 3 \mathrm{H}), 3.41-3.47(\mathrm{~m}, 1 \mathrm{H}), 3.57-3.63(\mathrm{~m}$, $1 \mathrm{H}), 3.76(\mathrm{~d}, J=12.3 \mathrm{~Hz}, 1 \mathrm{H}), 4.83-4.86(\mathrm{~m}, 1 \mathrm{H})$, 6.42 (s, 1H, HetH), 7.03-7.38 (m, 4H, ArH). Anal. Calcd. for $\mathrm{C}_{18} \mathrm{H}_{19} \mathrm{BrN}_{4} \mathrm{O}_{6} \mathrm{~S}: \mathrm{C}, 43.30 ; \mathrm{H}, 3.84 ; \mathrm{N}$, 11.22. Found: C, 43.48; H, 4.08; N, 3.52.

(3g) Yield 83\%. Mp 144-146 ${ }^{\circ} \mathrm{C} .[\alpha]_{\mathrm{D}}-73^{\circ}$ (c 1, $\mathrm{CH}_{3} \mathrm{OH}$ ). ${ }^{1} \mathrm{H}$ NMR $\left(300 \mathrm{MHz}, \mathrm{D}_{2} \mathrm{O}\right): \delta 2.33$ (s, 3H, Het- $\left.\mathrm{CH}_{3}\right)$, $3.21-3.37(\mathrm{~m}, 3 \mathrm{H}), 3.39-3.48(\mathrm{~m}, 1 \mathrm{H}), 3.52-3.61(\mathrm{~m}$, $1 \mathrm{H}), 3.67-3.75(\mathrm{~m}, 1 \mathrm{H}), 6.43$ (s, 1H, HetH), 6.95-7.63 (m, $4 \mathrm{H}, \mathrm{ArH})$. Anal. Calcd. for $\mathrm{C}_{18} \mathrm{H}_{19} \mathrm{ClN}_{4} \mathrm{O}_{6} \mathrm{~S}$ : C, 47.53; H, 4.21; N, 12.32. Found: C, 47.81; H, 4.36; N, 12.08 .

(3h) Yield 90\%. Mp 130-132 ${ }^{\circ} \mathrm{C} .[\alpha]_{\mathrm{D}}-44^{\circ}$ (c 1, $\mathrm{CH}_{3} \mathrm{OH}$ ). ${ }^{1} \mathrm{H}$ NMR $\left(300 \mathrm{MHz}, \mathrm{D}_{2} \mathrm{O}\right): \delta 2.35\left(\mathrm{~s}, 3 \mathrm{H}\right.$, Het- $\left.\mathrm{CH}_{3}\right)$, $3.30-3.34(\mathrm{~m}, 3 \mathrm{H}), 3.41-3.47(\mathrm{~m}, 1 \mathrm{H}), 3.55-3.61(\mathrm{~m}$, $1 \mathrm{H}), 3.74(\mathrm{~d}, J=11.1 \mathrm{~Hz}, 1 \mathrm{H}), 4.83(\mathrm{~d}, J=10.2 \mathrm{~Hz}$, $1 \mathrm{H}), 6.42$ (s, 1H, HetH), 7.02-7.44 (m, 4H, ArH). Anal. Calcd. for $\mathrm{C}_{18} \mathrm{H}_{19} \mathrm{ClN}_{4} \mathrm{O}_{6} \mathrm{~S}$ : C, 47.53; H, 4.21; $\mathrm{N}, 12.32$. Found: C, 47.65; H, 4.34; N, 12.10.

(3i) Yield 92\%. Mp $138-140{ }^{\circ} \mathrm{C} .[\alpha]_{\mathrm{D}}-40^{\circ}$ (c 1, $\mathrm{CH}_{3} \mathrm{OH}$ ). ${ }^{1} \mathrm{H}$ NMR $\left(300 \mathrm{MHz}, \mathrm{D}_{2} \mathrm{O}\right): \delta 2.35\left(\mathrm{~s}, 3 \mathrm{H}\right.$, Het- $\left.\mathrm{CH}_{3}\right)$, $3.28-3.36(\mathrm{~m}, 3 \mathrm{H}), 3.41-3.43(\mathrm{~m}, 1 \mathrm{H}), 3.56-3.62$ $(\mathrm{m}, 1 \mathrm{H}), 3.75(\mathrm{~d}, J=12.3 \mathrm{~Hz}, 1 \mathrm{H}), 6.42(\mathrm{~s}, 1 \mathrm{H}$, $\mathrm{HetH}), 7.02-7.44(\mathrm{~m}, 4 \mathrm{H}, \mathrm{ArH})$. Anal. Calcd. for $\mathrm{C}_{18} \mathrm{H}_{19} \mathrm{ClN}_{4} \mathrm{O}_{6} \mathrm{~S}: \mathrm{C}, 47.53 ; \mathrm{H}, 4.21 ; \mathrm{N}, 12.32$. Found: C, 47.33; H, 4.29; N, 12.02 .

\section{Results and Discussion}

5-Methylisoxazole-3-carbohydrazide, which is required as a starting material, was prepared according to the literature [16]. As shown in Scheme 1, 4-aryl-5-(5-methylisoxazol-3yl)-1,2,4-triazol-3-thiols (1a-1i) were prepared via the reaction of 5-methylisoxazole-3-carbohydrazide with arylisothiocyanates and then cyclization in the presence of $2 \mathrm{~mol} / \mathrm{L}$ aqueous potassium carbonate solution.

S- $\beta$-D-acetylglucosides $(\mathbf{2} \mathbf{a}-\mathbf{2} \mathbf{i})$ were obtained with the improved Koenigs-Knorr method. We use potassium hydroxide as the base to avoid the use of more expensive or toxic reagents as prometers, such as phase transfer catalyst $\left(\mathrm{BnEt}_{3} \mathrm{~N}^{+} \mathrm{Br}^{-}\right)$[18] or heavy metal salts $\left(\mathrm{Hg}(\mathrm{CN})_{2}\right)$ [13]. The coupling reaction of 4-aryl-5-(5-methylisoxazol3 -yl)-1,2,4-triazol-3-thiols with 2,3,4,6-tetra-O-acetyl- $\alpha$-Dglucopyranosyl bromide was conducted in ethanol in the presence of potassium hydroxide, and nine new S- $\beta$-Dacetylglucosides were afforded. The results showed that the reaction is a typical $\mathrm{S}_{\mathrm{N}} 2$ reaction and a convenient method to stereoselectively synthesis of only the single $\beta$-anomer.

The classical method of deacetylation of the sugar moiety employing a catalytic amount of sodium methoxide in methanol is described by Zemplén and Kuntz [19]. However, 
the Zemplén deacetylation is not suitable for the deprotection of carbohydrates containing 5-methylisoxazole-substituted 1,2,4-triazole due to the instability of the isoxazole ring under strong basic condition. Finally, the removal of the protecting groups was easily achieved by treatment with dry ammonia gas in dry methanol. The final desired S-glucosides containing 5-methylisoxazole-substituted 1,2,4-triazole (3a$3 i$ ) were successfully obtained in good yields.

The structures of $\mathbf{2} \mathbf{a}-\mathbf{2} \mathbf{i}$ and $\mathbf{3 a - 3} \mathbf{i}$ were confirmed by elemental analyses and spectral data. The ${ }^{1} \mathrm{H}$ NMR spectral data of compounds $\mathbf{2 a - 2 \mathbf { i }}$ showed the presence of four acetyl groups through the four singlets in the region of 1.94-2.05 ppm. In addition, seven hydrogen atoms of the sugar moiety were also observed in these spectra. They exhibited multiplets at $3.75-5.58 \mathrm{ppm}$. Only $\beta$-anomer was obtained as judged by a doublet at $\delta 5.52-5.58\left(J_{H 1, H 2}=\right.$ $9.9-10.8 \mathrm{~Hz})$ of the anomeric proton $(\mathrm{H}-1)$ in the sugar moiety. Compounds $\mathbf{2 a - 2 i}$ showed two singlets at $\delta 2.41-2.44$ and 6.54-6.64, which were assigned to the protons of methyl and isoxazole ring, respectively.

The ${ }^{1} \mathrm{H}$ NMR spectra of $\mathbf{3 a - 3 i}$ provided support to successful deacetylation reaction by the disappearance of the four sharp singlets in the region of 1.94-2.05 ppm as observed in the spectra of $\mathbf{2 a - 2} \mathbf{i}$. The aryl groups of compounds $\mathbf{2 a - 2} \mathbf{i}$ and $3 \mathbf{a}-3 \mathbf{i}$ were found in the region of $6.84-7.71 \mathrm{ppm}$. The other ${ }^{1} \mathrm{H}$ NMR spectral data was found approximately the same as observed in the case of the corresponding acetylated glucosides. The elemental analysis of these compounds was good agreement with calculated values.

Compounds $\mathbf{3 a - 3 i}$ were screened for their antibacterial activity against Escherichia coli and Staphylococcus aureus. The results showed that most of the compounds were inactive against these microorganisms. Further investigation on biological activities is in progress.

\section{Acknowledgment}

The authors gratefully acknowledge the financial support from Xinxiang Medical University.

\section{References}

[1] R. Romagnoli, P. G. Baraldi, O. Cruz-Lopez et al., "Synthesis and antitumor activity of 1,5-disubstituted 1,2,4-triazoles as cis-restricted combretastatin analogues," Journal of Medicinal Chemistry, vol. 53, no. 10, pp. 4248-4258, 2010.

[2] J. Chen, X. Y. Sun, K. Y. Chai et al., "Synthesis and anticonvulsant evaluation of 4-(4-alkoxylphenyl)-3-ethyl$4 \mathrm{H}-1,2,4$-triazoles as open-chain analogues of 7-alkoxyl4,5-dihydro[1,2,4] triazolo[4,3-a]quinolines," Bioorganic and Medicinal Chemistry, vol. 15, no. 21, pp. 6775-6781, 2007.

[3] J. Xu, Y. Cao, J. Zhang et al., "Design, synthesis and antifungal activities of novel 1,2,4-triazole derivatives," European Journal of Medicinal Chemistry, vol. 46, no. 7, pp. 3142-3148, 2011.

[4] Y. M. Ma, R. H. Liu, X. Y. Gong et al., "Synthesis and Herbicidal Activity of N,N-Diethyl-3-(arylselenonyl)-1H-1,2,4-triazole-1carboxamide," Journal of Agricultural and Food Chemistry, vol. 54, no. 20, pp. 7724-7728, 2006.
[5] X. P. Hui, L. M. Zhang, Z. Y. Zhang, Q. Wang, and F. Wang, "Synthesis and biological activity of 1,3,4-oxadiazole, 1,3,4thiadiazole and 1,2,4-triazole derivatives of 5-methylisoxazole," Indian Journal of Chemistry B, vol. 38, article 679, 1999.

[6] X. P. Hui, L. M. Zhang, Z. Y. Zhang, Q. Wang, and F. Wang, "Synthesis and antibacterial activity of s-Triazoles, sTriazolo[3,4-b]-1,3,4-thiadiazines and s-Triazolo[3,4-b]-1,3,4thiadiazoles of 5-Methylisoxazole," Journal of the Chinese Chemical Society, vol. 47, article 535, 2000.

[7] Y. L. Gao, G. L. Zhao, M. Liu, Y. L. Wang, W. R. Xu, and J. W. Wang, "Thiadiazole-based thioglycosides as sodiumglucose co-transporter 2 (SGLT2) inhibitors," Chinese Journal of Chemistry, vol. 28, no. 4, pp. 605-612, 2010.

[8] V. Liska, J. E. Dyr, J. Suttnar, I. Hirsch, and V. Vonka, "Production and simple purification of a protein encoded by part of the gag gene of HIV-1 in the Escherichia coli $\mathrm{HB}_{101 \mathrm{~F}}^{+}$ expression system inducible by lactose and isopropyl- $\beta$-dthiogalactopyranoside," Journal of Chromatography B, vol. 656, no. 1, pp. 127-133, 1994.

[9] J. T. Witkoski, R. K. Robins, R. W. Sidwell, and L. N. Simon, "Design, synthesis, and broad spectrum antiviral activity of 1-.beta.-D-ribofuranosyl-1,2,4-triazole-3-carboxamide and related nucleosides," Journal of Medicinal Chemistry, vol. 15, no. 11, pp. 1150-1154, 1972.

[10] R. A. Smith and W. Kirkpatrick, Eds., Ribavirin, A Broad Spectrum Antiviral Agent, Academic Press, New York, NY, USA, 1980.

[11] R. A. Smith, V. Knight, and J. A. D. Smith, Clinical Applications of Ribavirin, Academic Press, New York, NY, USA, 1984.

[12] G. D. Kini, R. K. Robins, and T. L. Avery, "Synthesis and antitumor activity of ribavirin imidates. New facile synthesis of ribavirin amidine (1-.beta.-D-ribofuranosyl-1,2,4-triazole3-carboxamidine hydrochloride)," Journal of Medicinal Chemistry, vol. 32, no. 7, pp. 1447-1449, 1989.

[13] K. Zamani, K. Faghihi, and R. Iqbal, "Synthesis and structure determination of some new N-glycosides of 4,5-disubstituted1,2,4-triazole-3-thiones," Journal of the Chinese Chemical Society, vol. 49, no. 6, pp. 1041-1044, 2002.

[14] N. A. Hassan, "Syntheses of acyclic C-glycosidic derivatives of 1,2,4-triazoles by cycloadditions of 1-aza-2-azoniaallene salts to D-glucononitrile-2,3,4,5,6-pentaacetate," Journal of Heterocyclic Chemistry, vol. 44, no. 4, pp. 933-936, 2007.

[15] W. A. El-Sayed, R. E. Abdel Megeid, and H. S. Abbas, "Synthesis and antimicrobial activity of new 1-[(tetrazol-5yl)methyl] indole derivatives, their 1,2,4-triazole thioglycosides and acyclic analogs," Archives of Pharmacal Research, vol. 34, no. 7, pp. 1085-1096, 2011.

[16] C. S. Marvel, "Studies on acyl thiosemicarbazides and related heterocycles (V) - syntheses of 1-isonicotinoyl-4-aryl thiosemicarbazides and related nitrogen, sulfur, oxygen 5-membered heterocycles," Organic Syntheses, vol. 1, article 238, 1951.

[17] Z. Y. Zhang, K. X. Yang, and F. L. Zeng, Chemical Journal of Chinese Universities, vol. 9, p. 239, 1988.

[18] Z. F. Wang, G. L. Zhao, W. Liu et al., "Design, synthesis and antidiabetic activity of thiadiazole-based thioglycosides as SGLT2 inhibitors," Chinese Journal of Organic Chemistry, vol. 30, no. 6, pp. 849-859, 2010.

[19] G. Zemplén and A. Kuntz, "Studien über Amygdalin, IV: Synthese des natürlichen 1-Amygdalins," Berichte der Deutschen Chemischen Gesellschaft B, vol. 57, no. 8, pp. 1357-1359, 1924. 

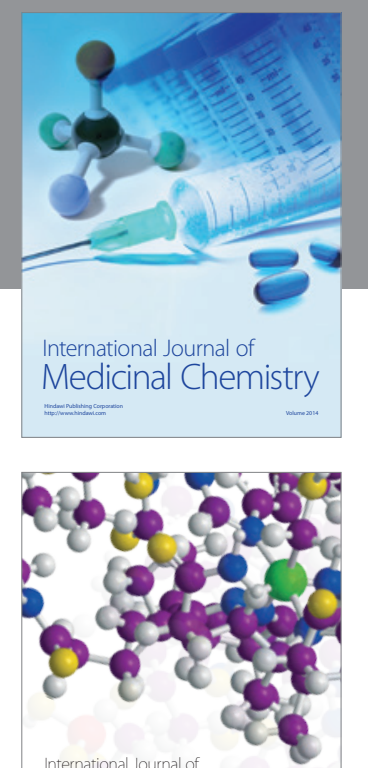

\section{Carbohydrate} Chemistry

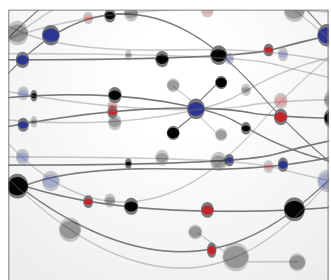

The Scientific World Journal
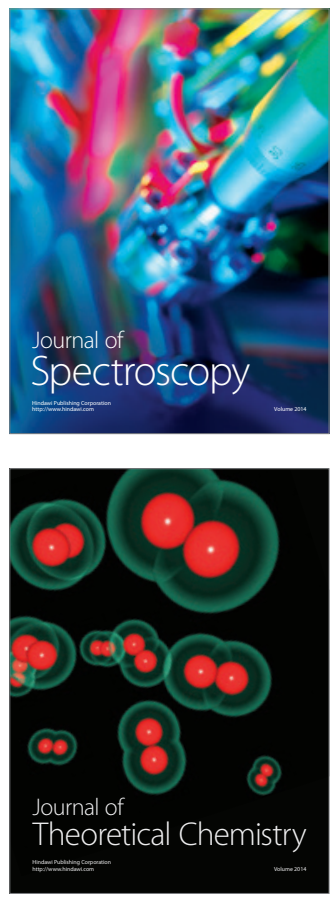
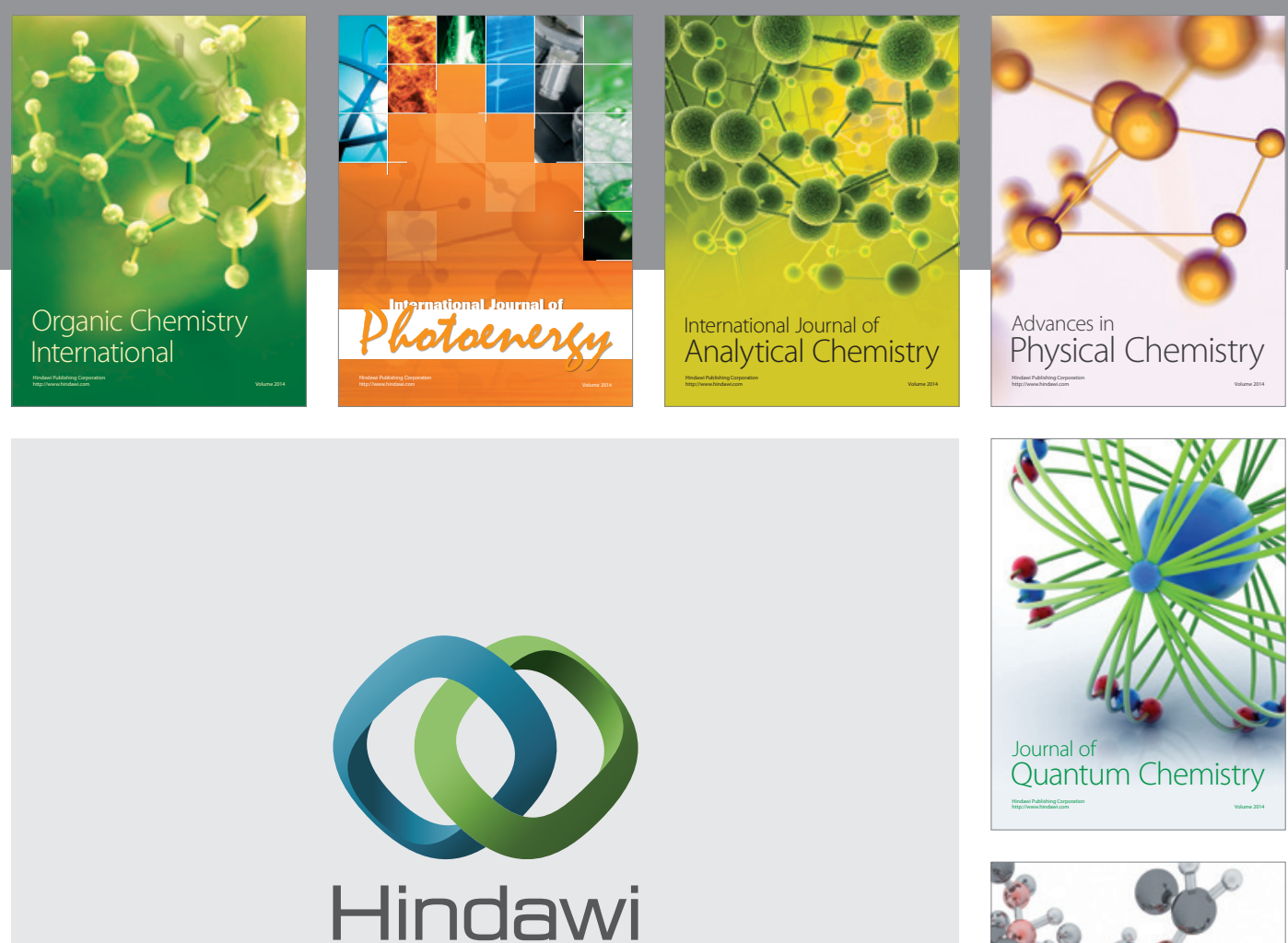

Submit your manuscripts at

http://www.hindawi.com

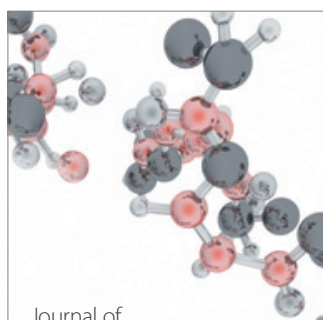

Analytical Methods

in Chemistry

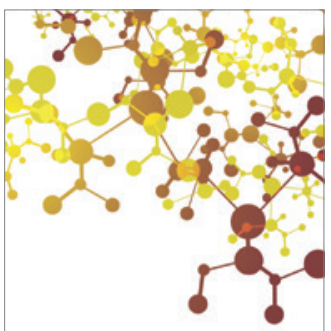

Journal of

Applied Chemistry

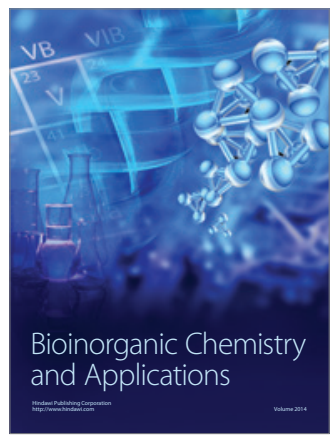

Inorganic Chemistry
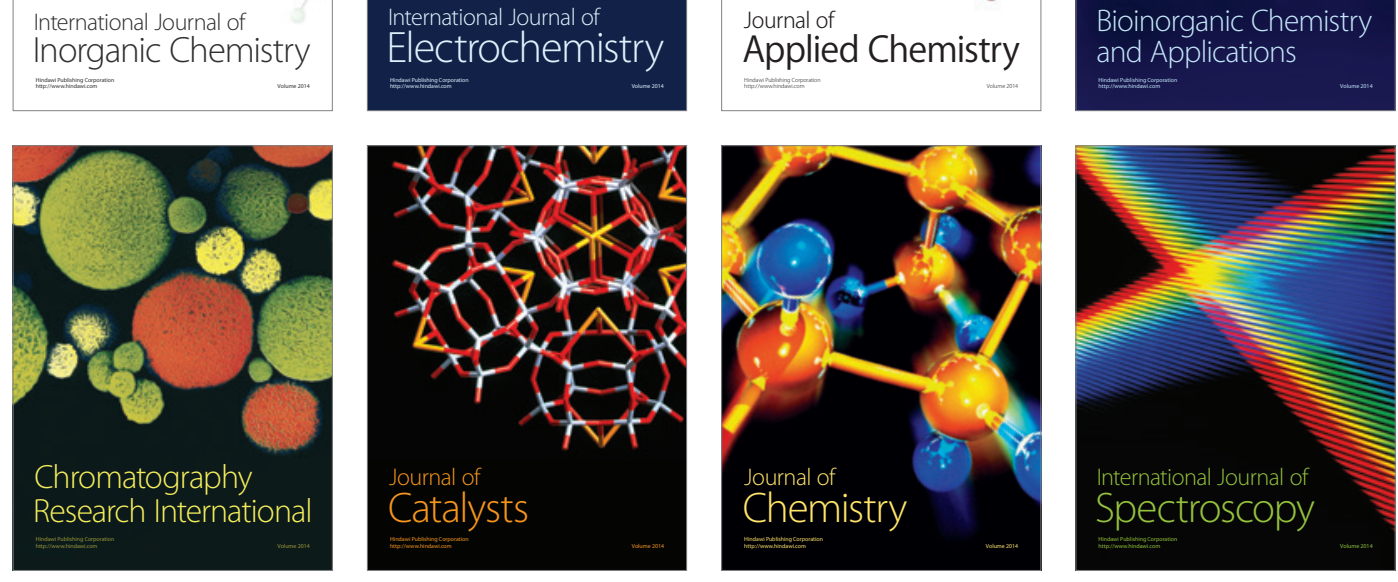